TRANSACTIONS OF THE

AMERICAN MATHEMATICAL SOCIETY

Volume 353, Number 11, Pages 4405-4427

S 0002-9947(01)02876-8

Article electronically published on July 9, 2001

\title{
A GEOMETRIC PARAMETRIZATION FOR THE VIRTUAL EULER CHARACTERISTICS OF THE MODULI SPACES OF REAL AND COMPLEX ALGEBRAIC CURVES
}

\author{
I. P. GOULDEN, J. L. HARER, AND D. M. JACKSON
}

\begin{abstract}
We determine an expression $\xi_{g}^{s}(\gamma)$ for the virtual Euler characteristics of the moduli spaces of $s$-pointed real $(\gamma=1 / 2)$ and complex $(\gamma=1)$ algebraic curves. In particular, for the space of real curves of genus $g$ with a fixed point free involution, we find that the Euler characteristic is $(-2)^{s-1}\left(1-2^{g-1}\right)(g+s-2) ! B_{g} / g$ ! where $B_{g}$ is the $g$ th Bernoulli number. This complements the result of Harer and Zagier that the Euler characteristic of the moduli space of complex algebraic curves is $(-1)^{s}(g+s-2) ! B_{g+1} /(g+1)(g-1)$ !

The proof uses Strebel differentials to triangulate the moduli spaces and some recent techniques for map enumeration to count cells. The approach involves a parameter $\gamma$ that permits specialization of the formula to the real and complex cases. This suggests that $\xi_{g}^{s}(\gamma)$ itself may describe the Euler characteristics of some related moduli spaces, although we do not yet know what these spaces might be.
\end{abstract}

\section{INTRODUCTION}

We show that the virtual Euler characteristics for the moduli spaces of $s$-pointed algebraic curves of genus $g$ can be determined from a polynomial $\xi_{g}^{s}(\gamma)$ in $\gamma^{-1}$ where the parameter $\gamma$ permits specialisation, through $\gamma=1$, to the complex case treated by Harer and Zagier and, through $\gamma=1 / 2$, to the real case. The latter yields a new result for the space of real curves of genus $g$ with a fixed point free involution, giving the Euler characteristic as $(-2)^{s-1}\left(1-2^{g-1}\right)(g+s-2) ! B_{g} / g$ ! where $B_{g}$ is the $g$ th Bernoulli number.

A detailed analysis of the parametrized quantity $\xi_{g}^{s}(\gamma)$ provides startling evidence that it has geometric significance. Indeed, it may in fact be the virtual Euler characteristic of some moduli spaces, as yet unidentified. We carry out this analysis by establishing a connection with a previous combinatorial conjecture that the indeterminate $b=\gamma^{-1}-1$ is associated with a combinatorial invariant for cell decompositions. The development uses Strebel differentials to triangulate the moduli spaces, and the identification of $\gamma$ both as a parameter in a Jack symmetric function through the irreducible characters of the symmetric group and the hyperoctohedral group, and as a parameter in a matrix model through generalized Selberg integrals. Both the matrix models of Hermitian and real symmetric matrices and these character theoretic formulations for counting cell decompositions are used.

To explain the parametrization that we have in mind, we begin with a brief introduction first to the complex case and then to the real case.

Received by the editors January 22, 1999 and, in revised form, April 7, 1999.

2000 Mathematics Subject Classification. Primary 58D29, 58C35; Secondary 05C30, 05E05. 
1.1. The complex and real cases. The moduli space $\mathcal{M}_{g}^{s}$ of $s$-pointed complex curves of genus $g$ has been an important object of study in many branches of mathematics for a long time. The global structure of these spaces is only partially understood; in particular, the cohomology of $\mathcal{M}_{g}^{s}$ is known in just a few cases. The primary tool for studying this cohomology has been a triangulation of $\mathcal{M}_{g}^{s}$ which is derived from work of Strebel on quadratic differentials by Harer [9]. In particular, the Euler characteristic of $\mathcal{M}_{g}^{s}$ was computed by Harer and Zagier [10] using this triangulation and an associated matrix model to count cells. Since that time, much work has been done in applying other matrix models and variants of the Strebel triangulation to the study of moduli spaces. This includes the alternative formulation by Penner [24] of the work in [10], the character-theoretic approach to the same work by Jackson [15, the exposition by Itzykson and Zuber [13], and most recently the beautiful work of Kontsevich [18] in establishing a conjecture of Witten [30] on intersection numbers of cohomology classes of $\overline{\mathcal{M}}_{g}^{s}$.

The moduli spaces of real algebraic curves have only come into the foreground more recently. Classically, a real algebraic curve $C$ is the set of simultaneous zeros in $\mathbb{R} P^{n}$ of a collection of homogeneous polynomials. By taking the solution in $\mathbb{C} P^{n}$ of the same set of polynomials, we can associate to the curve a Riemann surface and an antiholomorphic involution on it (induced by complex conjugation) whose fixed point set is the real curve $C$. This suggest defining a real curve as a pair consisting of a complex curve $X$ and an antiholomorphic involution $\tau$ and it is this point of view that we shall use here. For a fixed topological type of orientation reversing involution $\tau$, there is then a moduli space $\mathcal{M}_{g}^{s}(\tau)$ whose points are isomorphism classes of pairs of this form. Seppälä, Silhol, Buser and others [2, 26, 27] have made some progress in understanding these spaces, but up to now little is known about the topology of $\mathcal{M}_{g}^{s}(\tau)$. In the present work we will apply the technique of the Strebel differential to obtain a triangulation of $\mathcal{M}_{g}^{s}(\tau)$. We then count cells using enumerative techniques similar to those that have been used to study $\mathcal{M}_{g}^{s}$.

It is particularly startling that the complex and real cases can be treated simultaneously, through a generalized Selberg integral with parameter $\gamma$, and it is this that leads to the parametrization $\xi_{g}^{s}(\gamma)$ of the Euler characteristic.

1.2. The geometric parametrization. Central to our approach are the two generating series, $M^{O}$ for graphs embedded in orientable surfaces and $M$ for graphs embedded in locally orientable surfaces. Collectively, orientable and nonorientable surfaces are referred to as locally orientable. (Of course every surface is locally orientable, but the terminology is provided to make it clear that we are considering both orientable and non-orientable surfaces at once.) The embedded graphs are combinatorial objects and we will call them maps.

$M^{O}$ and $M$ have been given in integral form as evaluations of expectation operators over Hermitian complex matrices and real symmetric matrices. For orientable surfaces these are familiar from the work of 't Hooft [12] and Bessis, Itzykson and Zuber [1], while the corresponding result for surfaces is given by Goulden and Jackson [5]. In the particular case of specialisation to the virtual Euler characteristics of the moduli spaces, the diagonalized forms of the associated expectations have a common generalization $\xi_{g}^{s}(\gamma)$ as a generalized Selberg integral, whose evaluation is known [25. The virtual Euler characteristics for $\mathcal{M}_{g}^{s}$ and $\mathcal{M}_{g}^{s}(\tau)$ are therefore computed simultaneously by specialising the parameter, $\gamma$, that appears in this Selberg integral to $\gamma=1$ and $\gamma=1 / 2$, respectively. 
Character theoretic formulations for the generating series $M^{O}$ and $M$ have been given by Jackson and Visentin [16] and Goulden and Jackson [6] in terms of Schur functions and zonal polynomials, respectively. Both of these functions are specialisations of the Jack symmetric function through its parameter $\gamma$. We have conjectured [4] that, in the character theoretic setting, $b=\gamma^{-1}-1$ is an indeterminate associated with a combinatorial invariant of nonorientability for maps, and this identification is therefore inherited in the Selberg integral formulation of this paper. In the context of moduli spaces, this enables us therefore to conjecture that the parameter $b$ also has geometric significance, and that there is an appropriately defined moduli space involving $b$ that specializes to $\mathcal{M}_{g}^{s}$ and $\mathcal{M}_{g}^{s}(\tau)$ at $b=0$ and $b=1$, respectively.

Jack symmetric functions and their dependence on the parameter $\gamma$ have been studied quite intensively in the combinatorial community in the last decade (see, for example, Stanley [28. They also appear in connection 20] with the CalogeroSutherland model, for example, in statistical mechanics.

1.3. Organization of the paper. The paper is organized as follows. Section 2 gives the reduction of the calculation of the virtual Euler characteristic $\chi\left(\mathcal{M}_{g}^{s}(\tau)\right)$ to a combinatorial sum involving polygonal identifications. In Section 3 we show that both this combinatorial sum, and the corresponding sum for the complex case, can be realized as particular coefficients in the map series $M$ and $M^{O}$ at transformed arguments. We then evaluate these by first representing them each as matrix integrals and then constructing a single generalized Selberg integral $M_{\gamma}$, with parameter $\gamma$, that specializes to the diagonalized forms of these matrix integrals through choices of $\gamma$. This integral is evaluated in Section 4 through asymptotic properties of the gamma function to give the parametrized Euler characteristic, $\xi_{g}^{s}(\gamma)$, explicitly as a polynomial in $\gamma^{-1}$. In Section 5 we demonstrate that $M_{\gamma}$ can be reexpressed in terms of Jack symmetric functions with parameter $\gamma^{-1}$. A previous conjecture that gives combinatorial meaning to the parameter $b$ (recall that $b=\gamma^{-1}-1$ ) in the context of maps then leads to the conjecture that $b$ has geometric significance in the context of moduli spaces. For completeness, we include a brief account of the combinatorial encoding of maps and the algebras that carry the encoded combinatorial information that explain the appearance of Schur functions and zonal polynomials (which are Jack symmetric functions at $\gamma=1$ and $\gamma=1 / 2$, respectively).

The Appendix gives a table of the polynomial coefficients in $b$ that arise in the first few terms of $M_{\gamma}$. The polynomials are indexed by vertex distribution, the number of faces and the number of edges as combinatorial data. At $b=0$ and $b=1$ these evaluate to the numbers of maps, with prescribed combinatorial data, on orientable and locally orientable surfaces respectively.

\section{The MOdUl SPACES OF REAL CURVES}

2.1. The Teichmüller spaces and mapping class groups of nonorientable surfaces. By a real algebraic curve we will mean a pair $(X, \sigma)$ where $X$ is a Riemann surface and $\sigma$ is an antiholomorphic involution. Two such curves $\left(X_{1}, \sigma_{1}\right)$ and $\left(X_{2}, \sigma_{2}\right)$ are isomorphic if there is a biholomorphism $\phi: X \rightarrow Y$ such that $\phi \sigma_{1}=\sigma_{2} \phi$.

Fix a closed oriented surface $F$ of genus $g$ and let $\tau$ be an orientation reversing involution of $F$. The fixed point set $\operatorname{Fix}(\tau)$ of $\tau$ is a collection (possibly empty) 
of $m$ disjoint simple closed curves which separates $F$ into $\epsilon=1$ or 2 connected components. If $\tau_{1}$ is another such involution, $\tau$ is conjugate to $\tau_{1}$ if and only if $m$ $=m_{1}$ and $\epsilon=\epsilon_{1}$ (compare [26]). There are therefore $[(3 g+4) / 2]$ conjugacy classes of orientation reversing involutions on $F$. Fix $(\tau)$ is non-separating in $g+1$ cases, while in the remaining cases $\operatorname{Fix}(\tau)$ separates $F$ into two components, each of genus $(g-m+1) / 2$.

Choose $2 s$ distinct, ordered points $\left\{q_{i}\right\}$ on $F$ and let $\tau$ be an orientation reversing involution of $F$ which acts without fixed points on the $\left\{q_{i}\right\}$. We define the Teichmüller space $\mathcal{T}_{g}^{s}(\tau)$ to be the space of all isomorphism classes of quadruples $\left(X, \sigma,\left\{p_{i}\right\}, f\right)$ where $(X, \sigma)$ is a real algebraic curve, $\left\{p_{i}\right\}$ is an ordered collection of $2 s$ distinct points of $X$ on which $\sigma$ acts freely and

$$
f:\left(X,\left\{p_{i}\right\}\right) \rightarrow\left(F,\left\{q_{i}\right\}\right)
$$

is a homeomorphism preserving the ordering of the points such that $\tau f=f \sigma$. $(F$ is called a marking of $\left(X, \sigma,\left\{p_{i}\right\}\right)$.) The quadruples $\left(X, \sigma,\left\{p_{i}\right\}, f\right)$ and $\left(Y, \mu,\left\{r_{i}\right\}, g\right)$ are isomorphic if there exists an isomorphism $\phi$ from $(X, \sigma)$ to $(Y, \mu)$ with $\phi\left(p_{i}\right)=$ $r_{i}$ for each $i$ such that $g \phi$ is homotopic to $f$ rel $\left\{p_{i}\right\}$.

Since the automorphism group of any Riemann surface $X$ is finite, there are only finitely many antiholomorphic involutions $\sigma$ of $X$ (any two differ by an automorphism). The natural map $\mathcal{T}_{g}^{s}(\tau) \rightarrow \mathcal{T}_{g}^{2 s}$ which forgets the involution is therefore finite-to-one. Topologize $\mathcal{T}_{g}^{s}(\tau)$ via this map. By choosing an appropriate pair of pants decomposition of $F$, it is straightforward to use Fenchel-Nielsen coordinates to show that $\mathcal{T}_{g}^{s}(\tau)$ is homeomorphic to Euclidean space of dimension $3 g-3+2 s$. (Here $3 g-3$ dimensions come from the isomorphism class of the real curve while $2 s$ more dimensions come from the locations of $s$ of the points and these determine that of the others.)

It can be shown that when $\left(X, \sigma,\left\{p_{i}\right\}, f\right)$ is isomorphic to $\left(Y, \mu,\left\{r_{i}\right\}, g\right)$ using the definition above, the homotopy from $g \phi$ to $f$ can be replaced by an isotopy $H_{t}$ such that at each time $t, H_{t}\left(p_{i}\right)=q_{i}$ and $H_{t} \sigma=\tau H_{t}$. Therefore, if we let

$$
\pi_{X}: X \rightarrow X / \sigma
$$

and

$$
\pi_{F}: F \rightarrow F / \tau
$$

be the quotient maps, $\bar{p}_{i}=\pi\left(p_{i}\right)$ and $\bar{q}_{i}=\pi\left(q_{i}\right)$, the isomorphism class of $\left(X, \sigma,\left\{p_{i}\right\}\right.$, $f)$ is determined uniquely by $\left(X / \sigma,\left\{\bar{p}_{i}\right\}\right)$ and the homotopy class rel $\left\{\bar{p}_{i}\right\}$ of the homeomorphism of pairs $\left(X / \sigma,\left\{\bar{p}_{i}\right\}\right) \rightarrow\left(F / \tau,\left\{\bar{q}_{i}\right\}\right)$ induced by $f$. Thus $\mathcal{T}_{g}^{s}(\tau)$ may be thought of as the Teichmüller space of non-orientable surfaces of genus $g$ (Euler characteristic $1-g$ ) with $s$ ordered points and a local orientation of the surface at each point.

Next, recall that the mapping class group $\Gamma_{g}^{2 s}$ is the group of isotopy classes rel $\left\{q_{i}\right\}$ of self-homeomorphisms of $F$ that fix each $q_{i}$. We define the mapping class group $\Gamma_{g}^{s}(\tau)$ to be the subgroup of $\Gamma_{g}^{2 s}$ consisting of all mapping classes which admit a representative which commutes with $\tau$. It can be shown that two such representatives are homotopic rel $\left\{q_{i}\right\}$ if and only if they are isotopic via an isotopy which commutes with $\tau$ at each time. Since every self-homeomorphism of $F / \tau$ has a unique orientation preserving lift to $F$ which commutes with $\tau$ (the two lifts differ by $\tau$ which is orientation reversing), $\Gamma_{g}^{s}(\tau)$ may be identified with the group of isotopy classes $\left(\right.$ rel $\left.\left\{\bar{q}_{i}\right\}\right)$ of self-homeomorphisms of $F / \tau$ which preserve each point $\bar{q}_{i}$ and 
preserve a local orientation of $F / \tau$ at each $\pi\left(q_{i}\right)$. (Notice that unlike the usual mapping class group of a surface with boundary, we allow boundary components of $F / \tau$ to be permuted and we do not require that homeomorphisms or isotopies of homeomorphisms fix boundary curves pointwise.) The group $\Gamma_{g}^{s}(\tau)$ acts properly discontinuously on $\mathcal{T}_{g}^{s}(\tau)$ via the usual action of $\Gamma_{g}^{2 s}$ on $\mathcal{T}_{g}^{2 s}$. The quotient will be denoted $\mathcal{M}_{g}^{s}(\tau)$ and called the moduli space of $s$-pointed real algebraic curves. It is an orbifold (V-manifold) of real dimension $3 g-3+2 s$ and, like the moduli space of complex curves, it has a finite cover which is an ordinary manifold. When two involutions of $F$ are conjugate, there is a natural identification of one moduli space with the other, so there are $[(3 g+4) / 2]$ such moduli spaces for each $g$ and $s$.

2.2. The orbifold Euler characteristics. The Euler characteristic of an orbifold $\mathcal{M}$ which has a finite manifold branched covering $\widetilde{\mathcal{M}}$ of degree $d$ is defined to be

$$
\chi(\mathcal{M})=\frac{\chi(\widetilde{\mathcal{M}})}{d}
$$

where $\chi(\widetilde{\mathcal{M}})$ is the ordinary Euler characteristic of $\widetilde{\mathcal{M}}$. This number is independent of the choice of $\widetilde{\mathcal{M}}$ because Euler characteristics multiply by degree for unbranched coverings. Recall that the Euler characteristic of a group $G$ which is virtually torsion free is defined similarly,

$$
\chi(G)=\frac{\chi(\widetilde{G})}{d}
$$

where $\widetilde{G}$ is a torsion free subgroup of finite index $d$ in $G$. Since $\mathcal{T}_{g}^{s}(\tau)$ is contractible and $\Gamma_{g}^{s}(\tau)$ acts properly discontinuously on $\mathcal{T}_{g}^{s}(\tau)$ with quotient $\mathcal{M}_{g}^{s}(\tau)$,

$$
\chi\left(\mathcal{M}_{g}^{s}(\tau)\right)=\chi\left(\Gamma_{g}^{s}(\tau)\right) .
$$

2.3. The Euler characteristic for the real case. Let $\tau_{0}$ be an orientation reversing involution of $F$ without fixed points. Let $B_{g}$ be the $g$ th Bernoulli number, defined by the exponential generating series

$$
B(t)=\frac{t}{e^{t}-1}=\sum_{j \geq 0} B_{j} \frac{t^{j}}{j !} .
$$

Then the virtual Euler characteristic for the real case is given by the following result.

Theorem 2.1. For $g \geq 1, s \geq 0, g+s>1$,

$$
\chi\left(\mathcal{M}_{g}^{s}\left(\tau_{0}\right)\right)=(-2)^{s-1}\left(1-2^{g-1}\right) \frac{(g+s-2) !}{g !} B_{g} .
$$

From this theorem it is clear that $\chi\left(\mathcal{M}_{g}^{s}\left(\tau_{0}\right)\right)=0$ when $g$ is odd and $g+s>1$ since $B_{2 n+1}=0$ for $n \geq 1$. The group $\Gamma_{1}\left(\tau_{0}\right)$ is the subgroup of $\Gamma_{1}$ consisting of all maps which commute with $\tau_{0}$. Now $\Gamma_{1}$ is isomorphic to $S L_{2}(\mathbb{Z})$ since every element of $\Gamma_{1}$ is determined by the map it induces on $\pi_{1}\left(T^{2}\right)=\mathbb{Z} \oplus \mathbb{Z}$. The map $\tau_{0}$ induces

$$
\left(\begin{array}{rr}
-1 & 0 \\
0 & 1
\end{array}\right)
$$


on $\pi_{1}\left(T^{2}\right)$, so $\Gamma_{1}\left(\tau_{0}\right)$ is simply $\{ \pm I\}$. Since $\Gamma_{0}$ and $\Gamma_{0}^{2}$ are trivial, so are $\Gamma_{0}\left(\tau_{0}\right)$ and $\Gamma_{0}^{1}\left(\tau_{0}\right)$. Also, $\pi_{0}^{2}$ is infinite cyclic, isomorphic to $\Gamma_{0}^{2}\left(\tau_{0}\right)$. Therefore the above theorem may be completed with the statements:

$\chi\left(\mathcal{M}_{1}^{0}\left(\tau_{0}\right)\right)=\frac{1}{2}, \quad \chi\left(\mathcal{M}_{0}^{s}\left(\tau_{0}\right)\right)=1 \quad$ for $s=0$ or $1, \quad \chi\left(\mathcal{M}_{0}^{s}\left(\tau_{0}\right)\right)=0 \quad$ for all $s \geq 2$.

For all $g$ and $s$ with $g+s \geq 1$ there are exact sequences

$$
1 \rightarrow \pi_{g}^{s} \rightarrow \Gamma_{g}^{s+1}\left(\tau_{0}\right) \rightarrow \Gamma_{g}^{s}\left(\tau_{0}\right) \rightarrow 1
$$

where $\pi_{g}^{s}$ is the fundamental group of the orientable surface of genus $g$ with $2 s$ points removed. The map

$$
\Gamma_{g}^{s+1}\left(\tau_{0}\right) \rightarrow \Gamma_{g}^{s}\left(\tau_{0}\right)
$$

is obtained by forgetting one of the pairs of points, say $\left(q_{2 s+1}, q_{2 s+2}\right)$, which are interchanged by $\tau_{0}$. Its kernel is $\pi_{g}^{s}$ because the isotopy to the identity of an element of $\Gamma_{g}^{s+1}\left(\tau_{0}\right)$ which fixes $\left\{q_{1}, \ldots, q_{2 s}\right\}$ creates and is created by moving the point $q_{2 s+1}$ along a loop representing an element of the fundamental group of $F-\left\{q_{1}, \ldots, q_{2 s}\right\}$. ( $F$ occurs here instead of $F / \tau_{0}$ because the points $q_{i}$ are not permuted. Equivalently, the self-maps of $F / \tau$ which we consider are all locally orientable at the points $\pi\left(q_{i}\right)$, so only orientation preserving loops lie in $\Gamma_{g}^{s+1}\left(\tau_{0}\right)$.)

Now, given any short exact sequence of groups

$$
1 \rightarrow A \rightarrow B \rightarrow C \rightarrow 1
$$

we have

$$
\chi(B)=\chi(A) \chi(C) .
$$

This means that for each $g$, Theorem 2.1 only needs to be proven for one value of $s$. We will, in fact, show it for all $s \geq 1$, as this is the more natural approach. The short exact sequence is necessary, however, to establish the case where $s=0$.

For the involutions $\tau$ which have fixed points, let us consider separately the cases where the fixed point sets do or do not separate $F$. If $\operatorname{Fix}(\tau)$ consists of $m$ disjoint simple closed curves which together do not separate $F$, then $F / \tau$ is a non-orientable surface with $m$ boundary components. The Dehn twist on a boundary curve of $F / \tau$ is isotopic to the identity since it lifts to two Dehn twists on isotopic curves with opposite twist directions. In addition, elements can permute the components of $\operatorname{Fix}(\tau)$. Therefore $\Gamma_{g-m}^{s+m}\left(\tau_{0}\right)$ may be identified with a subgroup of index $m$ ! in the mapping class group of $\left(F / \tau,\left\{\pi\left(q_{i}\right)\right\}\right)$. The Euler characteristic of the former is given by Theorem 2.1 above, that of the latter is obtained by dividing by $m$ !.

If $\operatorname{Fix}(\tau)$ consists of $m$ disjoint simple closed curves which together do separate $F$, then $F / \tau$ is an orientable surface with $m$ boundary components of genus $h$, where $g=2 h+m-1$. In this case the ordinary mapping class group $\Gamma_{h}^{s+m}$ may be identified with a subgroup of index $m$ ! in the mapping class group of $\left(F / \tau,\left\{\pi\left(q_{i}\right)\right\}\right)$. The Euler characteristic of $\Gamma_{h}^{s+m}$ was computed in [10]. Combining all of these results we have the following general statement.

Corollary 2.2. If $\operatorname{Fix}(\tau)$ is non-separating and consists of $m$ simple closed curves, then

$$
\chi\left(\Gamma_{g}^{s}(\tau)\right)=(-2)^{s+m-1}\left(1-2^{g-m-1}\right) \frac{(g+s-2) !}{m !(g-m) !} B_{g-m} .
$$


If $\operatorname{Fix}(\tau)$ is separating and consists of $m$ simple closed curves, then $g-m+1$ must be even and

$$
\chi\left(\Gamma_{g}^{s}(\tau)\right)=(-1)^{s+m} \frac{(g-m+s-2) !}{m !(g-m+1)(g-m-1) !} B_{g-m+1} .
$$

2.4. Moduli spaces and polygonal identifications. We begin the proof of Theorem 2.1 by first expressing $\chi\left(\mathcal{M}_{g}^{s}\left(\tau_{0}\right)\right)$ in terms of pairings of sides of polygons. Recall [29] that for every $k$-pointed Riemann surface $\left(X,\left\{p_{1}, \ldots, p_{k}\right\}\right)$ and every collection of weights $\left(t_{1}, \ldots, t_{k}\right)$ with $t_{i}>0$ and $t_{1}+\ldots+t_{k}=1$, there is a unique quadratic differential which has double poles at the points $p_{i}$ of type $-d z^{2} / z^{2}$, no other poles, closed real trajectories and connected singular trajectories with the distance (in the norm defined by the quadratic differential) from $p_{i}$ to the singular trajectory equal to $t_{i}$. This concept was first used in [9] (see also [8]) to describe cells for the moduli space of curves. The singular trajectories of the differential then decompose $X$ into $k$ disks which, after scaling $X$, can be taken to be radius $t_{i}$, centered at each $p_{i}$. This exhibits $\left(X,\left\{p_{1}, \ldots, p_{k}\right\}\right)$ as obtained in a unique manner by identifying the sides of $k$ disks $D_{i}$ in $\mathbb{C}$ (compare [8] and [9]). Fixing an identification pattern with a marking and varying the edge lengths determines an open cell in $\mathcal{T}_{g}^{k} \times \Delta^{k-1}$, where $\Delta^{k-1}$ is a $k-1$ simplex.

Suppose now that $X$ admits a fixed point free involution $\tau_{0}$ which interchanges the points $p_{2 i-1}$ and $p_{2 i}$ for $i=1, \ldots, s$. It follows directly from Strebel's work that the real and imaginary trajectories of the differential are invariant under $\tau_{0}$. (This fact was first pointed out to us by Seppälä.) This means that $\mathcal{T}_{g}^{s}\left(\tau_{0}\right) \times \Delta^{s-1}$ admits an ideal triangulation with cells determined as follows: Choose positive integers $n_{1}, \ldots, n_{s}$ with $n_{1}+\ldots+n_{s}=2 n$. For each $i$ take two copies $P_{i}^{+}$and $P_{i}^{-}$ of a polygon with $n_{i}$ sides. Also take a pairing $\omega$ of the sides of $\bigcup_{1<i<s} P_{i}^{+} \cup P_{i}^{-}$ which gives an oriented, connected surface of genus $g$ and is compatible with the interchange maps $P_{i}^{+} \longleftrightarrow P_{i}^{-}$. These maps together induce an involution $\sigma_{\omega}$ of $\left(\bigcup_{1 \leq i \leq s} \partial P_{i}^{+} \cup \partial P_{i}^{-}\right) / \omega$. We assume that $\sigma_{\omega}$ is orientation reversing. This pairing (as in [9] and [10]) must satisfy the requirement that the valence of each vertex of the boundary graph $\left(\bigcup_{1 \leq i \leq s} \partial P_{i}^{+} \cup \partial P_{i}^{-}\right) / \omega$ is at least 3 . Finally, choose a marking $f_{\omega}$ of the result that identifies the center of $P_{i}^{+}$with $q_{2 i-1}$ and the center of $P_{i}^{-}$ with $q_{2 i}$. Varying the edge lengths determines an open cell whose dimension is $n-1$. Orbits of cells are determined by the identification alone (without the marking). This gives an ideal triangulation in the following sense: Each marked identification determines an open cell, corresponding to positive lengths on each edge. By allowing an edge length to go to 0 , we obtain a cell of one lower dimension as long as the resulting identification still gives a surface of genus $g$. If, however, the result has smaller genus, the face lies outside of $\mathcal{T}_{g}^{s}\left(\tau_{0}\right) \times \Delta^{s-1}$.

By forming the quotient of $X_{\omega}$ by $\tau_{0}$, the identification $\omega$ induces an identification $\omega^{\prime}$ of the union of $s$ polygons $P_{i}$ having $n_{i}$ edges to obtain a connected, nonorientable surface $X_{\omega}^{\prime}$ of Euler characteristic $1-g$. (The boundary graph has $n$ edges and $1+n-g-s$ vertices and the same valence condition as above, since $\tau_{0}$ is fixed point free.) The marking $f_{\omega}$ induces a marking $f_{\omega}^{\prime}$ of $X_{\omega}^{\prime}$ which pairs the ordered center points $\left\{\bar{p}_{1}, \ldots, \bar{p}_{s}\right\}$ with $\left\{\bar{q}_{1}, \ldots, \overline{q_{s}}\right\}$.

Conversely, given an identification $\omega^{\prime}$ of the edges of $\bigcup_{1 \leq i \leq s} P_{i}$ to obtain a nonorientable surface $X_{\omega}^{\prime}$ of Euler characteristic $1-g$, with special points $\left\{\bar{p}_{1}, \ldots, \bar{p}_{s}\right\}$, and given a marking $f_{\omega}^{\prime}$ of $X_{\omega}^{\prime}$ with $f_{\omega}^{\prime}\left(\bar{p}_{i}\right)=\bar{q}_{i}$, there are $2^{s-1}$ corresponding choices of the pairing $\omega$ and marking $f_{\omega}$. To see this, visualize the disks $P_{i}^{+}$and $P_{i}^{-}$in 
a common plane so that assigning $U$ for an up normal or $D$ for a down normal orients them. Fix $U$ as the orientation for $P_{1}^{+}$and $D$ for $P_{1}^{-}$. Arbitrarily assign $U$ or $D$ to $P_{i}^{+}$and the opposite to $P_{i}^{-}$for $i=2, \ldots, s$. Now there is one and only one identification $\omega$ lifting $\omega^{\prime}$ which gives a surface on which the chosen orientations agree.

Think of the disks $P_{i}$ as also lying in a common plane with a $U$ orientation on each. Let $e_{1}$ be an edge of $P_{i}$ and $e_{2}$ an edge of $P_{j}$ (perhaps $i=j$ ) and suppose that $\omega^{\prime}$ pairs $e_{1}$ with $e_{2}$. Let $e_{1}^{U}$ be the edge corresponding to $e_{1}$ in either $P_{i}^{+}$or $P_{i}^{-}$, whichever is labelled $U$. Similarly define $e_{1}^{D}, e_{2}^{U}$ and $e_{2}^{D}$. If $\omega^{\prime}$ pairs $e_{1}$ and $e_{2}$ so that the orientations on $P_{i}$ and $P_{j}$ agree, then $e_{1}^{U}$ is paired with $e_{2}^{U}$ and $e_{1}^{D}$ with $e_{2}^{D}$. Otherwise they switch. (Notice that we could have performed the entire process by first starting with any $P_{i}^{+}$as either up or down and we would have the same $2^{s-1}$ possibilities.) The marking $f_{\omega}^{\prime}$ lifts to a unique $f_{\omega}$ once we specify which point of $\pi^{-1}\left(\bar{q}_{1}\right)$ to call $q_{1}$. Therefore, given $\left(\omega^{\prime}, f_{\omega}^{\prime}\right)$, there are exactly $2^{s-1}$ distinct lifts $\left(\omega, f_{\omega}\right)$. Since elements of the mapping class group $\Gamma_{g}^{s}\left(\tau_{0}\right)$ do not permute the points $\left\{q_{i}\right\}$, there are similarly $2^{s-1}$ distinct $\omega$.

We now consider polygonal identifications induced by $\omega^{\prime}$. Define $\lambda_{g}^{N}\left(n_{1}, \ldots, n_{s}\right)$ to be the number of identifications $\omega$ of the edges of $\bigcup_{1 \leq i \leq s} P_{i}$ that give a nonorientable surface of genus $g$ (Euler characteristic $1-g$ ) and so that the valence of each vertex of the boundary graph $\left(\bigcup_{1 \leq i \leq s} \partial P_{i}\right) / \omega$ is at least 3 . Here we are assuming that an initial edge of each $P_{i}$ is fixed so that, for example when $s=1$, the identifications $a a b b$ and $a b b a$ would be counted as different ways of constructing a Klein bottle. As an illustration,

$$
\lambda_{1}^{N}(2)=4
$$

corresponding to the four patterns:

$$
a a b b, \quad a b b a, \quad a b a b^{-1}, \quad a b a^{-1} b .
$$

Notice that if the surface has Euler characteristic $1-g$, then the number of vertices of the boundary graph is $1+n-g-s$.

Similarly, we define $\lambda_{g}^{O}\left(n_{1}, \ldots, n_{s}\right)$ to be the number of identifications of the edges of $\bigcup_{1 \leq i \leq s} P_{i}$ that give a orientable surface of genus $g$ (Euler characteristic $2-2 g$ ) with the same valence condition. Finally, define $\lambda_{g}\left(n_{1}, \ldots, n_{s}\right)$ to be the number of identifications of the edges of $\bigcup_{1 \leq i \leq s} P_{i}$ that give a surface of Euler characteristic $1-g$, orientable or not, with the same valence condition.

Now set

$$
\lambda_{g}^{s}(n)=\sum_{n_{1}+\cdots+n_{s}=2 n} \lambda_{g}\left(n_{1}, \ldots, n_{s}\right),
$$

and let $\lambda_{g}^{s, N}(n)$ and $\lambda_{(g+1) / 2}^{s, O}(n)$ be the sums, over the same range, with summands $\lambda_{g}^{N}\left(n_{1}, \ldots, n_{s}\right)$ and $\lambda_{(g+1) / 2}^{O}\left(n_{1}, \ldots, n_{s}\right)$, respectively. Finally, let

$$
\Lambda_{g}^{s}=\sum_{n=g+s}^{3 g+3 s-3} \frac{(-1)^{n-s}}{2 n} \lambda_{g}^{s}(n)
$$

and let $\Lambda_{g}^{s, N}$ and $\Lambda_{(g+1) / 2}^{s, O}$ be the analogous sums in which $\lambda_{g}^{s}(n)$ is replaced by $\lambda_{g}^{s, N}(n)$ and $\lambda_{(g+1) / 2}^{s, O}(n)$, respectively.

Next we express $\chi\left(\mathcal{M}_{g}^{s}\left(\tau_{0}\right)\right)$ in terms of one of the combinatorial sums defined above. 
Lemma 2.3.

$$
\chi\left(\mathcal{M}_{g}^{s}\left(\tau_{0}\right)\right)=2^{s-1} \Lambda_{g}^{s, N} .
$$

Proof. Recall that $\left.\mathcal{M}_{g}^{s}\left(\tau_{0}\right)\right)$ is an orbifold and by definition, each point of an orbifold $\mathcal{M}$ has a neighborhood that is locally modeled on $\mathbb{R}^{\operatorname{dim}(\mathcal{M})}$ modulo a finite group. An orbifold triangulation of $\mathcal{M}$ is a triangulation by simplices $\omega$ which have the property that the finite group associated to each point of the interior of $\omega$ is the same. If we call this group $G(\omega)$, the Euler characteristic on $\mathcal{M}$ is computed with the formula

$$
\chi(\mathcal{M})=\sum_{\omega} \frac{(-1)^{\operatorname{dim}(\omega)}}{o(G(\omega))} .
$$

To compute the Euler characteristic of $\mathcal{M}_{g}^{s}\left(\tau_{0}\right)$, consider the ideal triangulation of $\mathcal{M}_{g}^{s}\left(\tau_{0}\right) \times \Delta^{s-1}$ described by identification patterns. We cannot apply the formula above directly because cells of the triangulation must be closed. Instead we use the dual complex described in [9]: $\mathcal{M}_{g}^{s}\left(\tau_{0}\right) \times \Delta^{s-1}$ retracts onto a spine complex $Y$ of dimension $2 g-3+3 s$ which has a $k$-cell for each identification pattern $\omega$ with $n-1=3 g-3 s-4-k$. The cells are the identification patterns and the local groups are the symmetries of the configuration which determines the cell. Since these must fix the points $q_{i}$, they are all cyclic. Counting each identification pattern of (with no initial edges) weighted by the reciprocal of the order of the cyclic symmetry group is the same as counting each pattern with a choice of initial edge weighted by $1 / 2 n$ (compare [10]). The result follows.

Now clearly

$$
\Lambda_{g}^{s}=\Lambda_{g}^{s, N}+\Lambda_{(g+1) / 2}^{s, O},
$$

where the second term on the right is naturally zero when $g$ is even. Then Lemma2.3 and (4) enable us to establish the result of Theorem 2.1 by determining $\Lambda_{g}^{s}$ and $\Lambda_{(g+1) / 2}^{s, O}$. This will be carried out in the next section, where we actually determine a parametrized quantity that specializes to each of $\Lambda_{g}^{s}$ and $\Lambda_{(g+1) / 2}^{s, O}$ at different values of the parameter.

Previously Harer and Zagier [10] showed that $\chi\left(\mathcal{M}_{g}^{s}\right)=\Lambda_{(g+1) / 2}^{s, O}$ and thus determined that

$$
\chi\left(\mathcal{M}_{g}^{s}\right)=(-1)^{s} \frac{(g+s-2) !}{(g+1)(g-1) !} B_{g+1},
$$

when $g$ is odd (of course, $\chi\left(\mathcal{M}_{g}^{s}\right)=0$ for $g$ even).

\section{Matrix models And the COUnting Series For EMBedded GraphS}

3.1. Polygonal identifications and graph embeddings. To determine $\Lambda_{g}^{s}$ and $\Lambda_{(g+1) / 2}^{s, O}$ we first identify $\lambda_{g}^{s}(n)$ and $\lambda_{(g+1) / 2}^{s, O}(n)$ as solutions of equivalent enumerative questions for combinatorial maps, since appropriate forms for their generating series are already available, from current work in algebraic combinatorics. A map is an embedded graph in a surface with the property that each complementary region is a 2-cell. In topology one says the graph fills the surface and in combinatorics ones calls this a 2-cell embedding. The graph divides the surface into regions called faces. In an orientable surface a map is rooted by distinguishing an edge, and an end of this edge. In nonorientable surfaces an edge is distinguished together with 
an end of this edge, and a side of this end of the edge. Rooted maps have only the trivial automorphism. From this point of view, an identification $\omega$ of the edges of $P_{n}$ with boundary graph $\left(\partial P_{n}\right) / \omega$ corresponds precisely to a two-cell embedding of the boundary graph with a single face; the rooting allows us to uniquely recover the labels of the sides and the orientation of $P_{n}$. Thus we have the following combinatorial identifications:

- $\lambda_{g}^{s, O}(n)$ is $s$ ! times the number of rooted maps with s faces, $n$ edges and no vertices of valences 1 or 2 , in orientable surfaces of genus $g$ (Euler characteristic $2-2 g)$,

- $\lambda_{g}^{s}(n)$ is $s$ ! times the number of rooted maps with s faces, $n$ edges and no vertices of valences 1 or 2 , in locally orientable surfaces of Euler characteristic $1-g$.

For $\mathrm{i}=\left(i_{1}, i_{2}, \ldots\right)$, let $m(\mathrm{i}, j, n)$ and $m^{O}(\mathrm{i}, j, n)$ be, respectively, the numbers of rooted maps in locally orientable and orientable surfaces, with $n$ edges, $j$ faces and $i_{k}$ vertices of valence $k$ for $k \geq 1$. Thus $\sum_{k \geq 1} k i_{k}=2 n$, and $\sum_{k \geq 1} i_{k}=|V|$, the number of vertices. But from the Euler-Poincaré theorem, for the maps counted by $\lambda_{(g+1) / 2}^{O}(n)$ and $\lambda_{g}(n)$ we have $|F|-|E|+|V|=s-n+|V|=1-g$ so $|V|=n-g-s+1$. Thus we can write

$$
\lambda_{g}^{s}(n)=s ! \sum_{\mathrm{i}} m(\mathrm{i}, s, n), \quad \lambda_{(g+1) / 2}^{s, O}(n)=s ! \sum_{\mathrm{i}} m^{O}(\mathrm{i}, s, n),
$$

where, in both cases the sum is over i such that

$$
i_{1}=i_{2}=0, \quad \sum_{k \geq 3} k i_{k}=2 n, \quad \sum_{k \geq 3} i_{k}=n-g-s+1 .
$$

The vector $\mathrm{i}$ is called the vertex distribution of such maps. Let the generating series for maps with respect to vertex distribution be

$$
M(\mathrm{y}, x, z)=\sum_{\mathrm{i}, j, n} m(\mathrm{i}, j, n) \mathrm{y}^{\mathrm{i}} x^{j} z^{n}, \quad M^{O}(\mathrm{y}, x, z)=\sum_{\mathrm{i}, j, n} m^{O}(\mathrm{i}, j, n) \mathrm{y}^{\mathrm{i}} x^{j} z^{n},
$$

where $\mathrm{y}=\left(y_{1}, y_{2}, \ldots\right)$, and $\mathrm{y}^{\mathrm{i}}=\prod_{k \geq 1} y_{k}^{i_{k}}$.

3.2. A pair of matrix integrals. These two series have the following integral representations, as given in Jackson [14], for $M^{O}$, and Goulden and Jackson [5], for $M:$

$$
\begin{aligned}
M^{O}(\mathrm{y}, N, z) & =2 z \frac{\partial}{\partial z} \log \left(\frac{\int_{\mathcal{V}_{N}} e^{\sum_{k \geq 1} \frac{1}{k} y_{k} \sqrt{z}^{k} \text { trace } \mathrm{M}^{k}} e^{-\frac{1}{2} \operatorname{trace} \mathrm{M}^{2}} d \mathrm{M}}{\int_{\mathcal{V}_{N}} e^{-\frac{1}{2} \mathrm{trace} \mathrm{M}^{2}} d \mathrm{M}}\right), \\
M(\mathrm{y}, N, z) & =4 z \frac{\partial}{\partial z} \log \left(\frac{\int_{\mathcal{W}_{N}} e^{\frac{1}{2} \sum_{k \geq 1} \frac{1}{k} y_{k} \sqrt{z}^{k} \text { trace } \mathrm{M}^{k}} e^{-\frac{1}{4} \mathrm{trace}^{2}} d \mathrm{M}}{\int_{\mathcal{W}_{N}} e^{-\frac{1}{4} \mathrm{trace} \mathrm{M}^{2}} d \mathrm{M}}\right),
\end{aligned}
$$

where $\mathcal{V}_{N}$ and $\mathcal{W}_{N}$ are, respectively, the vector spaces of Hermitian complex and real symmetric $N \times N$ matrices. The reader who wishes to have greater detail is directed to the above sources, and to the discussion in Section 5 of this paper.

The integrals over $\mathcal{V}_{N}$ and $\mathcal{W}_{N}$ can be transformed by the Weyl integration theorems [11] to the following integrals over the reals. For a positive integer $N$, let 
$\lambda=\left(\lambda_{1}, \ldots, \lambda_{N}\right)$, let $V(\lambda)$ be the Vandermonde determinant $\prod_{1 \leq i<j \leq N}\left(\lambda_{j}-\lambda_{i}\right)$, let $p_{k}(\lambda)=\lambda_{1}^{k}+\cdots+\lambda_{N}^{k}$, the power sum symmetric function of degree $k$, for $k \geq 1$, and $d \lambda=d \lambda_{1} \cdots d \lambda_{N}$. Then

$M^{O}(\mathrm{y}, N, z)=2 z \frac{\partial}{\partial z} \log \left(\frac{\int_{\mathbb{R}^{N}}|V(\lambda)|^{2} e^{\sum_{k \geq 1} \frac{1}{k} \sqrt{z}^{k} y_{k} p_{k}(\lambda)} e^{-\frac{1}{2} p_{2}(\lambda)} d \lambda}{\int_{\mathbb{R}^{N}}|V(\lambda)|^{2} e^{-\frac{1}{2} p_{2}(\lambda)} d \lambda}\right)$

$M(\mathrm{y}, N, z)=4 z \frac{\partial}{\partial z} \log \left(\frac{\int_{\mathbb{R}^{N}}|V(\lambda)| e^{\frac{1}{2} \sum_{k \geq 1} \frac{1}{k} \sqrt{z}^{k} y_{k} p_{k}(\lambda)} e^{-\frac{1}{4} p_{2}(\lambda)} d \lambda}{\int_{\mathbb{R}^{N}}|V(\lambda)| e^{-\frac{1}{4} p_{2}(\lambda)} d \lambda}\right)$

A joint generalization of these is afforded by

$$
M_{\gamma}(\mathrm{y}, x, z)=\frac{2}{\gamma} z \frac{\partial}{\partial z} \log Z_{\gamma}(\mathrm{y}, x, z)
$$

where

$$
Z_{\gamma}(\mathrm{y}, N, z)=\frac{\int_{\mathbb{R}^{N}}|V(\lambda)|^{2 \gamma} e^{\gamma \sum_{k \geq 1} \frac{1}{k} \sqrt{z}^{k} y_{k} p_{k}(\lambda)} e^{-\frac{1}{2} \gamma p_{2}(\lambda)} d \lambda}{\int_{\mathbb{R}^{N}}|V(\lambda)|^{2 \gamma} e^{-\frac{1}{2} \gamma p_{2}(\lambda)} d \lambda},
$$

since

$$
M(\mathrm{y}, N, z)=M_{1 / 2}(\mathrm{y}, N, z), \quad M^{O}(\mathrm{y}, N, z)=M_{1}(\mathrm{y}, N, z) .
$$

Thus both $M$ and $M^{O}$ can be obtained by specializing the parameter $\gamma$ in $M_{\gamma}$, and it is the series $M_{\gamma}$ that we therefore study in detail. We now make a few comments about the interpretation of the above series. First, the numerator of (11) is an even function of $\sqrt{z}$ (to see this, consider the simultaneous substitutions $\left.\lambda_{j} \rightarrow-\lambda_{j}, j=1, \ldots, N\right)$, so the occurrence of $\sqrt{z}$ is of no concern; $M_{\gamma}$ really is a power series in $z$. Second, the second argument in these map series is an indeterminate, $x$, yet in each case the integral representation evaluates the series at any positive integer value $N$ (which is the dimension of the region of integration). However, the number of rooted maps with $n$ edges is finite for each $n$, so $M_{\gamma}$ can be viewed as a power series in $z$ with coefficients that are polynomials in $y_{1}, \ldots, y_{2 n}, x$. Thus, since this holds for infinitely many $N$, a polynomiality argument allows us to obtain $M_{\gamma}(\mathrm{y}, x, z)$ from $M_{\gamma}(\mathrm{y}, N, z)$, in principle, by deriving an explicit presentation of $M_{\gamma}(\mathrm{y}, N, z)$ as a power series in $z$, and formally replacing $N$ by $x$ in the resulting (polynomial) coefficients of each power of $z$.

In the following result, we obtain preliminary expressions for $\Lambda_{g}^{s}$ and $\Lambda_{(g+1) / 2}^{s, O}$, as specializations of the parameter in a quantity $\xi_{g}^{s}(\gamma)$, which is therefore referred to as the parametrized Euler characteristic. Here we use the notation $[A] B$ for the coefficient of $A$ in the expansion of $B$. The result gives $\xi_{g}^{s}(\gamma)$ as a particular coefficient of $M_{\gamma}$ evaluated at transformed arguments.

Proposition 3.1. Let

$$
\xi_{g}^{s}(\gamma)=s !(-1)^{s}\left[x^{s} t^{g+s-1}\right] \frac{1}{\gamma} \log W_{\gamma}(x, t),
$$


where

$$
W_{\gamma}(N, t)=\frac{\int_{\mathbb{R}^{N}}|V(\lambda)|^{2 \gamma}\left(\prod_{j=1}^{N} e^{-i \gamma \lambda_{j} / \sqrt{t}}\left(1-i \sqrt{t} \lambda_{j}\right)^{-\gamma / t}\right) d \lambda}{\int_{\mathbb{R}^{N}}|V(\lambda)|^{2 \gamma} e^{-\gamma p_{2}(\lambda) / 2} d \lambda} .
$$

Then, for $s, g \geq 1$,

$$
\Lambda_{g}^{s}=\xi_{g}^{s}\left(\frac{1}{2}\right), \quad \Lambda_{(g+1) / 2}^{s, O}=\xi_{g}^{s}(1) .
$$

Proof. Let $\mathbf{u}(t)=\left(u_{1}, u_{2}, \ldots\right)$, where $u_{1}=u_{2}=0, u_{k}=-(i \sqrt{t})^{k-2}, k \geq 3$ (here $i$ is $\sqrt{-1}$, and is not an index), and let $\Psi$ be the operator whose action is defined by

$$
\Psi f(\mathrm{y}, x, z)=\frac{1}{2} \int_{0}^{1} f(\mathbf{u}(t), x, z) \frac{d z}{z} .
$$

Now, for i satisfying the conditions in (6), under the specialization $\mathrm{y}=\mathbf{u}(t)$, we obtain

$$
\mathrm{y}^{\mathrm{i}}=(-1)^{n-g-s+1+\frac{1}{2}\{2 n-2(n-g-s+1)\}} t^{\frac{1}{2}\{2 n-2(n-g-s+1)\}}=(-1)^{n} t^{g+s-1} .
$$

Then we obtain $\Lambda_{g}^{s}=s !(-1)^{s}\left[x^{s} t^{g+s-1}\right] \Psi M$ and $\Lambda_{(g+1) / 2}^{s, O}=s !(-1)^{s}\left[x^{s} t^{g+s-1}\right] \Psi M^{O}$, for $g \geq 1, s \geq 1$, from (3), (5) and (7). But, from (10) and (11),

$$
\Psi M_{\gamma}=\frac{1}{\gamma} \int_{0}^{1}\left(\frac{\partial}{\partial z} \log Z_{\gamma}(\mathrm{u}(t), N, z)\right) d z=\frac{1}{\gamma} \log Z_{\gamma}(\mathrm{u}(t), N, 1),
$$

since $Z_{\gamma}(\mathrm{u}(t), N, 0)=1$. Now let $W_{\gamma}(N, t)=Z_{\gamma}(\mathrm{u}(t), N, 1)$. The result follows from (12) and the simplification

$$
\begin{aligned}
-\frac{1}{2} p_{2}(\lambda)+\sum_{k \geq 1} \frac{1}{k} u_{k} p_{k}(\lambda) & =-\sum_{k \geq 2} \frac{(i \sqrt{t})^{k-2}}{k} p_{k}(\lambda) \\
& =\frac{1}{i \sqrt{t}} p_{1}(\lambda)+\frac{1}{t} \sum_{k \geq 1} \frac{(i \sqrt{t})^{k}}{k} p_{k}(\lambda) \\
& =\sum_{j=1}^{N}\left(\frac{-i \lambda_{j}}{\sqrt{t}}+\frac{1}{t} \log \left(1-i \sqrt{t} \lambda_{j}\right)^{-1}\right) .
\end{aligned}
$$

Note that the application of $\Psi$ to $M_{\gamma}$ is justified, since the number of maps with no vertices of valences 1 or 2 (this is forced by the specialization $y_{1}=y_{2}=0$ ), and $|E|-|V|=g+s-1$ is finite for each $g$ and $s$ (e.g., these conditions imply that there are at most $3 g+3 s-3$ edges, as in the upper bound for the summation in (3)). Thus $W_{\gamma}(x, t)$ can be viewed as a power series in $t$ with coefficients that are polynomials in $x$.

\section{Determination of the parametrized Euler Characteristic}

The following detailed calculation involving properties of the gamma function of the parameter $\gamma$ is necessary since we wish to obtain the parametrized Euler characteristic $\xi_{g}^{s}(\gamma)$ as an explicit polynomial in $\gamma^{-1}$. In Section 5 we shall show that $b=\gamma^{-1}-1$ is an indeterminate that is conjectured to be associated with a combinatorial invariant of rooted maps. 
4.1. Selberg integration. We now determine $W_{\gamma}(x, t)$ from the integral representation (14) for $W_{\gamma}(N, t)$ by giving an explicit presentation of $W_{\gamma}(N, t)$ as a series in $t$, with coefficients that are polynomials in $N$, and then, appealing to polynomiality, formally replacing $N$ by $x$. To evaluate $W_{\gamma}(N, t)$ we use the following theorem due to Mehta 22. It is derived in a similar fashion to Selberg's theorem, but based on an integral of Cauchy instead of the beta integral (note that again $i=\sqrt{-1}$, and is not an index).

Theorem 4.1. For $\Re a, \Re b, \Re \alpha, \Re \beta>0, \Re(\alpha+\beta)>1$ and

$$
-\frac{1}{N}<\Re \gamma<\min \left(\frac{\Re \alpha}{N-1}, \frac{\Re \beta}{N-1}, \frac{\Re(\alpha+\beta+1)}{2(N-1)}\right),
$$

we have

$$
\begin{gathered}
\int_{\mathbb{R}^{N}}|V(\lambda)|^{2 \gamma} \prod_{j=1}^{N}\left(1+i \frac{\lambda_{j}}{a}\right)^{-\alpha}\left(1-i \frac{\lambda_{j}}{b}\right)^{-\beta} d \lambda \\
=\kappa \prod_{j=0}^{N-1} \frac{\Gamma(\alpha+\beta-(N+j-1) \gamma-1)}{\Gamma(\alpha-\gamma j) \Gamma(\beta-\gamma j)}
\end{gathered}
$$

where

$$
\kappa=\left(\frac{2 \pi a^{\alpha} b^{\beta}}{(a+b)^{(\alpha+\beta)-\gamma(N-1)-1}}\right)^{N} \prod_{j=0}^{N-1} \frac{\Gamma(1+(1+j) \gamma)}{\Gamma(1+\gamma)} .
$$

In proving the following result we use Mehta's integration theorem to evaluate $\log W_{\gamma}(N, t)$, and then apply the polynomiality argument to give an explicit (asymptotic) power series presentation of $\log W_{\gamma}(x, t)$.

Theorem 4.2. As an asymptotic expansion in $1 / t$ we have

$$
\begin{aligned}
& \log W_{\gamma}(x, t)=-\gamma x \sum_{k \geq 1} \frac{B_{2 k} t^{2 k-1}}{2 k(2 k-1)}+\sum_{\delta \geq 1} \frac{t^{\delta}}{\delta(\delta+1)} \sum_{r=1}^{\delta+1}\left(\begin{array}{c}
\delta+1 \\
r
\end{array}\right) B_{\delta+1-r} \\
& \times\left(\frac{x^{r}(-1)^{\delta+1-r}}{\gamma^{\delta-r}}-\sum_{m=1}^{r+1}\left(\begin{array}{c}
r+1 \\
m
\end{array}\right) \frac{B_{r+1-m}}{r+1} \frac{x^{m}}{\gamma^{r-m}}(-1)^{\delta-m}\right) .
\end{aligned}
$$

Proof. From (14),$W_{\gamma}(x, t)=F_{\gamma}(x, t) / G_{\gamma}(x, t)$, where

$$
F_{\gamma}(N, t)=\int_{\mathbb{R}^{N}}|V(\lambda)|^{2 \gamma}\left(\prod_{j=1}^{N} e^{-i \gamma \lambda_{j} / \sqrt{t}}\left(1-i \sqrt{t} \lambda_{j}\right)^{-\gamma / t}\right) d \lambda,
$$

and

$$
G_{\gamma}(N, t)=\int_{\mathbb{R}^{N}}|V(\lambda)|^{2 \gamma} e^{-\gamma p_{2}(\lambda) / 2} d \lambda
$$

We begin by determining the integrals $F_{\gamma}(N, t)$ and $G_{\gamma}(N, t)$ as different specializations of Mehta's integral in Theorem 4.1 above.

To determine $G_{\gamma}$ : We can rewrite $G_{\gamma}$ in the form

$$
G_{\gamma}(N, t)=\lim _{L \rightarrow \infty} \int_{\mathbb{R}^{N}}|V(\lambda)|^{2 \gamma} \prod_{j=1}^{N}\left(1+i \frac{\lambda_{j}}{\sqrt{L}}\right)^{-\frac{1}{2} \gamma L}\left(1-i \frac{\lambda_{j}}{\sqrt{L}}\right)^{-\frac{1}{2} \gamma L} d \lambda,
$$


so applying Theorem 4.1, with $a=b=\sqrt{L}$ and $\alpha=\beta=\gamma L / 2$, gives

$$
\begin{aligned}
G_{\gamma}= & \left(\prod_{j=0}^{N-1} \frac{\Gamma(1+(1+j) \gamma)}{\Gamma(1+\gamma)}\right) \lim _{L \rightarrow \infty}\left(\frac{2 \pi \sqrt{L}^{\gamma L}}{(2 \sqrt{L})^{\gamma L-\gamma(N-1)-1}}\right)^{N} \\
& \times \prod_{j=0}^{N-1} \frac{\Gamma(\gamma L-(N+j-1) \gamma-1)}{\Gamma\left(\frac{1}{2} \gamma L-\gamma j\right)^{2}} .
\end{aligned}
$$

But the duplication theorem for the gamma function gives

$$
\begin{aligned}
& \Gamma(\gamma L-(N+j-1) \gamma-1) \\
& =\frac{1}{\sqrt{\pi}} 2^{\gamma L-\gamma(N+j-1)-2} \Gamma\left(\frac{1}{2}(\gamma L-(N+j-1) \gamma-1)\right) \Gamma\left(\frac{1}{2}(\gamma L-(N+j-1) \gamma)\right),
\end{aligned}
$$

and it is straightforward to establish by standard properties of the gamma function that

$$
\begin{aligned}
& \lim _{L \rightarrow \infty} \frac{\Gamma\left(\frac{1}{2}(\gamma L-(N+j-1) \gamma-1)\right)}{\Gamma\left(\frac{1}{2} \gamma L-\gamma j\right)\left(\frac{1}{2} \gamma L\right)^{-\frac{1}{2}(N-j-1) \gamma-\frac{1}{2}}}=1 \\
& \quad=\lim _{L \rightarrow \infty} \frac{\Gamma\left(\frac{1}{2}(\gamma L-(N+j-1) \gamma)\right)}{\Gamma\left(\frac{1}{2} \gamma L-\gamma j\right)\left(\frac{1}{2} \gamma L\right)^{-\frac{1}{2}(N-j-1) \gamma}} .
\end{aligned}
$$

It follows that

$$
G_{\gamma}(N, t)=\left(\frac{\sqrt{2 \pi}}{\gamma^{\frac{1}{2}((N-1) \gamma+1)}}\right)^{N} \prod_{j=0}^{N-1} \frac{\Gamma(1+(1+j) \gamma)}{\Gamma(1+\gamma)} .
$$

To determine $F_{\gamma}$ : We can rewrite $F_{\gamma}$ in the form

$$
F_{\gamma}(N, t)=\lim _{L \rightarrow \infty} \int_{\mathbb{R}^{N}}|V(\lambda)|^{2 \gamma} \prod_{j=1}^{N}\left(1+i \frac{\sqrt{t}}{L} \lambda_{j}\right)^{-\gamma L / t}\left(1-i \sqrt{t} \lambda_{j}\right)^{-\gamma / t} d \lambda
$$

so applying Theorem 4.1 with $a=L / \sqrt{t}, b=1 / \sqrt{t}, \alpha=\gamma L / t$ and $\beta=\gamma / t$, gives

$$
F_{\gamma}=\left(\frac{2 \pi}{\sqrt{t}^{\gamma(N-1)+1}}\right)^{N} A_{\gamma}(N, t) B_{\gamma}(N, t) \prod_{j=0}^{N-1} \frac{\Gamma(1+(1+j) \gamma)}{\Gamma(1+\gamma) \Gamma\left(\frac{\gamma}{t}-\gamma j\right)},
$$

where

$$
A_{\gamma}(N, t)=\lim _{L \rightarrow \infty}\left(\frac{L^{\frac{\gamma L}{t}}\left(\frac{\gamma L}{t}\right)^{\frac{\gamma}{t}-\gamma(N-1)-1}}{(L+1)^{\frac{\gamma}{t}(L+1)-\gamma(N-1)-1}}\right)^{N}=\left(\left(\frac{\gamma}{t}\right)^{\frac{\gamma}{t}-\gamma(N-1)-1} e^{-\frac{\gamma}{t}}\right)^{N}
$$

and, again by standard properties of the gamma function,

$$
B_{\gamma}(N, t)=\lim _{L \rightarrow \infty} \prod_{j=0}^{N-1}\left(\frac{\Gamma\left(\frac{\gamma}{t}(L+1)-(N+j-1) \gamma-1\right)}{\Gamma\left(\frac{\gamma L}{t}-\gamma j\right)\left(\frac{\gamma L}{t}\right)^{\frac{\gamma}{t}-\gamma(N-1)-1}}\right)=1
$$


Thus

$$
F_{\gamma}(N, t)=\left(\frac{2 \pi}{t^{\frac{1}{2}(\gamma(N-1)+1)}} e^{-\gamma / t}\left(\frac{\gamma}{t}\right)^{\frac{\gamma}{t}-(\gamma(N-1)+1)}\right)^{N} \prod_{j=0}^{N-1} \frac{\Gamma(1+(1+j) \gamma)}{\Gamma(1+\gamma) \Gamma\left(\frac{\gamma}{t}-\gamma j\right)} .
$$

These evaluations for $F_{\gamma}$ and $G_{\gamma}$ immediately yield

$$
\begin{aligned}
W_{\gamma}(N, t) & =\frac{F_{\gamma}(N, t)}{G_{\gamma}(N, t)} \\
& =\left(\sqrt{2 \pi} e^{-\gamma / t}\left(\frac{\gamma}{t}\right)^{\frac{\gamma}{t}-\frac{1}{2}((N-1) \gamma+1)}\right)^{N} \prod_{j=0}^{N-1} \frac{1}{\Gamma\left(\frac{\gamma}{t}-\gamma j\right)} .
\end{aligned}
$$

We now exhibit $\log W_{\gamma}(N, t)$ explicitly as a power series in $t$, with coefficients that are polynomials in $N$. Let $N=q K$ and $\gamma=1 / q$ where $q$ and $K$ are positive integers. This specialization of $N$ does not affect the recoverability of $\log W_{\gamma}(x, t)$ from $\log W_{\gamma}(N, t)$, since there is an infinite set of such $K$, so the polynomiality argument still holds. With these choices for $N$ and $\gamma$ we have

$$
\prod_{j=0}^{q K-1} \Gamma\left(\frac{1}{t q}-\frac{j}{q}\right)=\frac{\Gamma\left(\frac{1}{t q}\right)}{\Gamma\left(\frac{1}{t q}-K\right)} \prod_{j=1}^{K}\left(\prod_{k=0}^{q-1} \Gamma\left(\frac{1}{t q}-j+\frac{k}{q}\right)\right),
$$

and the product over $k$ can be simplified by Gauss' multiplication theorem for the gamma function, whence

$$
\prod_{j=0}^{q K-1} \Gamma\left(\frac{1}{t q}-\frac{j}{q}\right)=\frac{\Gamma\left(\frac{1}{t q}\right)}{\Gamma\left(\frac{1}{t q}-K\right)}\left(\frac{\sqrt{2 \pi}^{q-1}}{q^{\frac{1}{t}-\frac{1}{2}-q(K+1)}}\right)^{K} \prod_{j=1}^{K} \Gamma\left(\frac{1}{t}-q j\right)
$$

But

$$
\prod_{j=1}^{K} \Gamma\left(\frac{1}{t}-q j\right)=\frac{\Gamma\left(\frac{1}{t}\right)^{K} t^{q K(K+1) / 2}}{\prod_{j=1}^{K} \prod_{l=1}^{q j}(1-l t)}, \quad \text { and } \quad \frac{\Gamma\left(\frac{1}{t q}\right)}{\Gamma\left(\frac{1}{t q}-K\right)}=\frac{1}{(t q)^{K}} \prod_{j=1}^{K}(1-t q j) .
$$

Combining these results with (17) and then with (16) gives

$$
W_{\frac{1}{q}}(q K, t)=\left(\frac{\sqrt{2 \pi t}}{\Gamma\left(\frac{1}{t}\right)(e t)^{\frac{1}{t}}}\right)^{K} \frac{\prod_{l=1}^{K} \prod_{j=1}^{q l}(1-j t)}{\prod_{j=1}^{K}(1-t q j)} .
$$

For the Bernoulli numbers $B_{j}$ given by (1), recall that

$$
\log \left(\frac{\Gamma\left(\frac{1}{t}\right)(e t)^{\frac{1}{t}}}{\sqrt{2 \pi t}}\right)=\sum_{k \geq 1} \frac{B_{2 k} t^{2 k-1}}{2 k(2 k-1)}
$$

as an asymptotic series in $1 / t$, and

$$
\sum_{j=1}^{n} j^{k}=\frac{1}{k+1} \sum_{r=1}^{k+1}\left(\begin{array}{c}
k+1 \\
r
\end{array}\right) B_{k+1-r}(-1)^{k+1-r} n^{r},
$$


for $k \geq 0$ and $n \geq 1$. Then applying these results to (18) gives, as an asymptotic expansion in $1 / t$,

$$
\begin{aligned}
\log W_{\frac{1}{q}}(q K, t)= & -K \sum_{k \geq 1} \frac{B_{2 k} t^{2 k-1}}{2 k(2 k-1)}+\sum_{j=1}^{K} \sum_{\delta \geq 1} \frac{t^{\delta}}{\delta} q^{\delta} j^{\delta}-\sum_{l=1}^{K} \sum_{j=1}^{q l} \sum_{\delta \geq 1} \frac{t^{\delta}}{\delta} j^{\delta} \\
= & -K \sum_{k \geq 1} \frac{B_{2 k} t^{2 k-1}}{2 k(2 k-1)}+\sum_{\delta \geq 1} \frac{t^{\delta}}{\delta(\delta+1)} \sum_{r=1}^{\delta+1}\left(\begin{array}{c}
\delta+1 \\
r
\end{array}\right) B_{\delta+1-r}(-1)^{\delta+1-r} \\
& \times\left(q^{\delta} K^{r}-\frac{q^{r}}{r+1} \sum_{m=1}^{r+1}\left(\begin{array}{c}
r+1 \\
m
\end{array}\right) B_{r+1-m}(-1)^{r+1-m} K^{m}\right)
\end{aligned}
$$

The result follows by polynomiality, upon replacing $q$ by $1 / \gamma$ and $K$ by $N \gamma$, thus giving an explicit power series presentation of $\log W_{\gamma}(x, t)$.

We are now in a position to give an explicit expression for the coefficient $\xi_{g}^{s}(\gamma)$ given in (13).

Corollary 4.3. For $g \geq 1, s \geq 1$,

$$
= \begin{cases}\frac{(g+s-2) !}{g !}(-1)^{s} \frac{B_{g}}{2}\left(\frac{1}{\gamma^{g}}-\frac{1}{\gamma}\right), & \text { if } g \text { is even, } \\
\frac{(g+s-2) !(-1)^{s+1}}{(g+1) !}\left\{\frac{(g+1) B_{g}}{\gamma^{g}}+\sum_{r=0}^{g+1}\left(\begin{array}{c}
g+1 \\
r
\end{array}\right) B_{g+1-r} \frac{B_{r}}{\gamma^{r}}\right\}, & \text { if } g \text { is odd. }\end{cases}
$$

Proof. The ring of asymptotic series in $1 / t$ is a subring of the ring of formal power series in $t$. Thus $\log W_{\gamma}$ has an asymptotic expansion that is the generating series for $\xi_{g}^{s}(\gamma)$ in $1 / t$. Moreover, if a function has an asymptotic series then it is unique. Thus from Proposition 3.1 and Theorem 4.2 we obtain, for $g \geq 1$ and $s \geq 2$,

$$
\begin{aligned}
& \xi_{g}^{s}(\gamma)=s !(-1)^{s}\left[x^{s} t^{g+s-1}\right] \frac{1}{\gamma} \log W_{\gamma}(x, t) \\
& =\frac{s !(-1)^{g+s}}{(g+s)(g+s-1)}\left\{\left(\begin{array}{c}
g+s \\
s
\end{array}\right) \frac{B_{g}}{\gamma^{g}}+\sum_{r=1}^{g+s}\left(\begin{array}{c}
g+s \\
r
\end{array}\right) B_{g+s-r}\left(\begin{array}{c}
r+1 \\
s
\end{array}\right) \frac{B_{r+1-s}}{(r+1) \gamma^{r+1-s}}\right\} \\
& =\frac{(g+s-2) !(-1)^{g+s}}{(g+1) !}\left\{\frac{(g+1) B_{g}}{\gamma^{g}}+\sum_{r=1}^{g+s}\left(\begin{array}{c}
g+1 \\
r+1-s
\end{array}\right) B_{g+s-r} \frac{B_{r+1-s}}{\gamma^{r+1-s}}\right\} .
\end{aligned}
$$

For $g \geq 1$ and $s=1$, we obtain

$$
\xi_{g}^{s}(\gamma)=\frac{(-1)^{g+1}}{g(g+1)}\left\{B_{g+1}+\frac{(g+1) B_{g}}{\gamma^{g}}+\sum_{r=1}^{g+1}\left(\begin{array}{c}
g+1 \\
r
\end{array}\right) B_{g+1-r} \frac{B_{r}}{\gamma^{r}}\right\}
$$

where, for the first term in (19), we have used the fact that the Bernoulli number $B_{j}$ is 0 for $j$ odd and greater than 1 .

Thus, for all $g \geq 1, s \geq 1$, we have

$$
\xi_{g}^{s}(\gamma)=\frac{(g+s-2) !(-1)^{g+s}}{(g+1) !}\left\{\frac{(g+1) B_{g}}{\gamma^{g}}+\sum_{r=0}^{g+s}\left(\begin{array}{c}
g+1 \\
r+1-s
\end{array}\right) B_{g+s-r} \frac{B_{r+1-s}}{\gamma^{r+1-s}}\right\}
$$


(For $s \geq 2$, the summand in (20) corresponding to $r=0$ is zero, so (20) agrees with (19) in this case. For $s=1$, the summand in (20) corresponding to $r=0$ is $B_{g+1}$, since $B_{0}=1$, so (20) agrees with (19) in this case also.) This gives the result immediately for $g$ odd.

For $g$ even, the zero values for Bernoulli numbers of odd subscript bigger than 1 mean that for the summation in (20), only the terms corresponding to $r=s$ and $r=g+s-1$ are non-zero, so for $g$ even we obtain

$$
\xi_{g}^{s}(\gamma)=\frac{(g+s-2) !(-1)^{s}}{(g+1) !}\left(\frac{(g+1) B_{g}}{\gamma^{g}}+(g+1) B_{g} B_{1} \frac{1}{\gamma}+(g+1) B_{1} B_{g} \frac{1}{\gamma^{g}}\right)
$$

and the result follows in this case, since $B_{1}=-1 / 2$.

4.2. Proof of Theorem [2.1. Next we prove Theorem 2.1 that gives $\Lambda_{g}^{s}$ and $\Lambda_{(g+1) / 2}^{s, O}$ by specializing the parameter $\gamma$ in Corollary 4.3. This is straightforward, although it requires additional properties of the Bernoulli numbers.

Corollary 4.4. For $g, s \geq 1$,

$$
\begin{aligned}
& \Lambda_{g}^{s}=\left\{\begin{array}{cc}
\frac{(g+s-2) !}{g !}(-1)^{s}\left(2^{g-1}-1\right) B_{g}, & \text { if } g \text { is even, } \\
\frac{(g+s-2) !(-1)^{s}}{(g+1)(g-1) !} B_{g+1}, & \text { if } g \text { is odd; }
\end{array}\right. \\
& \Lambda_{(g+1) / 2}^{s, O}=\left\{\begin{array}{cc}
0, & \text { if } g \text { is even }, \\
\frac{(g+s-2) !(-1)^{s}}{(g+1)(g-1) !} B_{g+1}, & \text { if } g \text { is odd. }
\end{array}\right.
\end{aligned}
$$

Proof. From Proposition 3.1 we have $\Lambda_{g}^{s}=\xi_{g}^{s}\left(\frac{1}{2}\right)$ and $\Lambda_{(g+1) / 2}^{s, O}=\xi_{g}^{s}(1)$, so for $g$ even the result follows immediately from Corollary 4.3 ,

For $g$ odd, we obtain from Corollary 4.3 that

$$
\xi_{g}^{s}(\gamma)=\frac{(g+s-2) !(-1)^{s+1}}{(g+1) !}\left(\frac{(g+1) B_{g}}{\gamma^{g}}+\left[\frac{t^{g+1}}{(g+1) !}\right] B(t) B\left(\frac{t}{\gamma}\right)\right),
$$

where $B(t)$ is the exponential generating series for Bernoulli numbers, given in (1). Now the following differential equations can be easily verified:

$$
\begin{aligned}
B(t)^{2} & =(1-t) B(t)-t \frac{t}{d t} B(t), \\
B(t) B(2 t) & =(1-t) B(t)-t \frac{t}{d t} B(t)-\frac{t}{2} B(2 t) .
\end{aligned}
$$

Thus (for $g$ odd), applying the first of these differential equations to (21) gives

$$
\begin{aligned}
\xi_{g}^{s}(1) & =\frac{(g+s-2) !(-1)^{s+1}}{(g+1) !}\left\{(g+1) B_{g}+\left(B_{g+1}-(g+1) B_{g}-(g+1) B_{g+1}\right)\right\} \\
& =\frac{(g+s-2) !(-1)^{s}}{(g+1)(g-1) !} B_{g+1} .
\end{aligned}
$$


Applying the second differential equation gives

$$
\begin{aligned}
\xi_{g}^{s}\left(\frac{1}{2}\right)= & \frac{(g+s-2) !(-1)^{s+1}}{(g+1) !}\left\{(g+1) B_{g} 2^{g}+\left(B_{g+1}-(g+1) B_{g}\right.\right. \\
& \left.\left.-(g+1) B_{g+1}-(g+1) B_{g} 2^{g-1}\right)\right\} \\
= & \frac{(g+s-2) !(-1)^{s+1}}{(g+1) !}\left\{-g B_{g+1}+(g+1) B_{g}\left(2^{g}-2^{g-1}-1\right)\right\},
\end{aligned}
$$

and the result follows, since $B_{g}=0$ for $g$ odd except when $g=1$, and $2^{g}-2^{g-1}-1=$ 0 when $g=1$.

As discussed at the end of Section 2, Corollary 4.4 completes the proof of Theorem 2.1 Note that the natural zero value for $\Lambda_{(g+1) / 2}^{s, O}$ for $g$ even is in agreement with the computed value for $\xi_{g}^{s}(1)$ in Corollary 4.4 .

\section{A geometric parametrization of the virtual Euler characteristic}

The argument $\gamma$ of the parametrized Euler characteristic $\xi_{g}^{s}(\gamma)$ was introduced as an artifact for interpolating between complex curves $(\gamma=1)$ and real curves $(\gamma=1 / 2)$, as specialized in (15). However, we conjecture that the parameter $\gamma$ itself has geometric significance. In particular, $\xi_{g}^{s}(\gamma)$ has been expressed as a polynomial in $1 / \gamma$ in Corollary 4.3 , and we conjecture that the coefficients in this polynomial have a geometric interpretation.

The evidence for this is indirect, but can be lifted from a combinatorial treatment of maps in Goulden and Jackson [4], that specializes to the series expansion (compare with (7)),

$$
M_{\gamma}(\mathrm{y}, x, z)=\sum_{\mathrm{i}, j, n} m_{\gamma}(\mathrm{i}, j, n) \mathrm{y}^{\mathrm{i}} x^{j} z^{n},
$$

of $M_{\gamma}(\mathrm{y}, x, z)$, defined in terms of an integral in (10). Note that $M_{\gamma}(\mathrm{y}, x, z)$ is in fact a parametrized map series because of the specializations in (12). We conjecture that $m_{\gamma}(\mathrm{i}, j, n)$ is a polynomial in $1 / \gamma$, with integer coefficients, and that these coefficients have combinatorial significance. Moreover,

$$
\xi_{g}^{s}(\gamma)=s !(-1)^{s}\left[x^{s} t^{g+s-1}\right] \Psi M_{\gamma},
$$

from the proof of Proposition 3.1, so we deduce that $\xi_{g}^{s}(\gamma)$ is a finite alternating summation of $m_{\gamma}(\mathrm{i}, j, n)$ 's, and thus we can lift the investigation of the significance of $m_{\gamma}(\mathrm{i}, j, n)$ to $\xi_{g}^{s}(\gamma)$ itself.

The lifting from maps is through properties of symmetric functions, and Jack functions in particular, which we now summarize (for further details see Macdonald [21]).

5.1. Jack symmetric functions. We say that $\mu=\left(\mu_{1}, \ldots, \mu_{k}\right)$ is a partition of $n$, written $\mu \vdash n$ or $|\mu|=n$, with $k$ parts, written $l(\mu)=k$, if $\mu_{1} \geq \ldots \geq \mu_{k} \geq 1$, and $\mu_{1}+\cdots+\mu_{k}=n$. The $\mu_{i}$ 's are the parts of $\mu$. The monomial symmetric function $m_{\mu}$ in a countable set of algebraically independent indeterminates $\mathrm{x}=\left(x_{1}, \ldots\right)$, is the sum of all distinct monomials in the $x_{i}$ 's whose exponents are the parts of $\mu$, with repetition, in some permuted order. If $\mathcal{P}$ is the set of all partitions (including a single, empty, partition of 0 ), then $\left\{m_{\mu}\right\}_{\mu \in \mathcal{P}}$ forms a basis for the ring of symmetric functions. If $p_{\mu}=p_{\mu_{1}} \cdots p_{\mu_{k}}$, where $p_{m}$ is the $m$ th power sum (symmetric function) 
in $\mathrm{x}$, then $\left\{p_{\mu}\right\}_{\mu \in \mathcal{P}}$ is also a basis, and hence we can define an inner product $\langle\quad, \quad\rangle_{\alpha}$ for an indeterminate $\alpha$, by the orthogonality

$$
\left\langle p_{\lambda}, p_{\mu}\right\rangle_{\alpha}=\frac{|\mu| !}{\left|\mathcal{C}_{\mu}\right|} \alpha^{l(\mu)} \delta_{\lambda, \mu},
$$

where $\mathcal{C}_{\mu}$ is the conjugacy class in the symmetric group on $|\mu|$ symbols with disjoint cycle lengths given by the parts of $\mu$. Then with respect to this inner product, the Jack symmetric functions $\left\{J_{\mu}(\mathrm{x} ; \alpha)\right\}_{\mu \in \mathcal{P}}$ also form a basis for symmetric functions in $\mathrm{x}$ (depending on the parameter $\alpha$ ), defined by the conditions

$$
\begin{gathered}
\left\langle J_{\lambda}, J_{\mu}\right\rangle_{\alpha}=0 \text { for } \lambda \neq \mu ; \quad\left[m_{\mu}\right] J_{\lambda}=0 \text { unless } \mu \preceq \lambda ; \\
{\left[x_{1} \cdots x_{n}\right] J_{\mu}=n !, \text { where }|\mu|=n,}
\end{gathered}
$$

imposing orthogonality, triangularity and normalization, respectively, where $\preceq$ denotes reverse lexicographic order. The Cauchy theorem for Jack functions is

$$
\prod_{i, j \geq 1}\left(1-x_{i} y_{j}\right)^{-1 / \alpha}=\sum_{\theta \in \mathcal{P}} \frac{J_{\theta}(\mathrm{x} ; \alpha) J_{\theta}(\mathrm{y} ; \alpha)}{\left\langle J_{\theta}, J_{\theta}\right\rangle_{\alpha}} .
$$

Finally, let

$$
\langle f(\lambda)\rangle_{\mathbb{R}^{N}}=\frac{\int_{\mathbb{R}^{N}}|V(\lambda)|^{2 \gamma} e^{-\frac{\gamma}{2} p_{2}} f(\lambda) d \lambda}{\int_{\mathbb{R}^{N}}|V(\lambda)|^{2 \gamma} e^{-\frac{\gamma}{2} p_{2}} d \lambda} .
$$

Then, as a connection between integration and Jack symmetric functions, it has been previously conjectured in Goulden and Jackson [5] that

$$
\left\langle J_{\theta}(\lambda ; \alpha)\right\rangle_{\mathbb{R}^{N}}=J_{\theta}\left(1_{N} ; \alpha\right)\left[p_{2}^{m}\right] J_{\theta},
$$

where $1_{N}$ is the vector with $N 1$ 's, and $\theta \vdash 2 m$. This conjecture was recently proved by Okounkov [23].

5.2. Lifting from the combinatorial conjecture. We can now give a symmetric function representation for $M_{\gamma}(\mathbf{p}(\mathrm{y}), x, z)$.

Proposition 5.1. Let $\mathbf{p}(\mathrm{y})=\left(p_{1}(\mathrm{y}), \ldots\right)$. Then

$$
M_{\gamma}(\mathbf{p}(\mathrm{y}), x, z)=\frac{2}{\gamma} z \frac{\partial}{\partial z} \log \sum_{\theta \in \mathcal{P}} z^{\frac{|\theta|}{2}} \frac{J_{\theta}(\mathrm{y} ; 1 / \gamma) J_{\theta}\left(1_{N} ; 1 / \gamma\right)}{\left\langle J_{\theta}, J_{\theta}\right\rangle_{1 / \gamma}}\left[p_{2}^{\frac{|\theta|}{2}}\right] J_{\theta} .
$$

Proof. If we replace $y_{i}$ by $p_{i}(\mathrm{y}), i \geq 1$ (this presents no difficulties since the $p_{i}(\mathrm{y})$ are algebraically independent for countable y), then from (10) and (11) we obtain

$$
\begin{aligned}
M_{\gamma}(\mathbf{p}(\mathrm{y}), x, z) & =\frac{2}{\gamma} z \frac{\partial}{\partial z} \log \left\langle e^{\gamma \sum_{k \geq 1} \frac{1}{k} \sqrt{z}^{k} p_{k}(\mathrm{y}) p_{k}(\lambda)}\right\rangle_{\mathbb{R}^{N}} \\
& =\frac{2}{\gamma} z \frac{\partial}{\partial z} \log \left\langle\prod_{i \geq 1}^{N} \prod_{j=1}^{N}\left(1-\sqrt{z} y_{i} \lambda_{j}\right)^{-\gamma}\right\rangle_{\mathbb{R}^{N}} \\
& =\frac{2}{\gamma} z \frac{\partial}{\partial z} \log \left\langle\sum_{\theta \in \mathcal{P}} z^{\frac{|\theta|}{2}} \frac{J_{\theta}(\mathrm{y} ; 1 / \gamma) J_{\theta}(\lambda ; 1 / \gamma)}{\left\langle J_{\theta}, J_{\theta}\right\rangle_{1 / \gamma}}\right\rangle_{\mathbb{R}^{N}},
\end{aligned}
$$

from Cauchy's theorem above, and the result follows from the integral evaluation (23). 
Jack function series like that on the right hand side of (24) have been considered in [4], where a combinatorial conjecture is made about their coefficients. The conjecture specialized to $m_{\gamma}(\mathrm{i}, j, n)$ is that $m_{1 /(b+1)}(\mathrm{i}, j, n)$ is a polynomial in $b$ with nonnegative integer coefficients. Note from (71), (12) and (22) that these coefficients sum to $m(\mathrm{i}, j, n)$ (here $b=1$ ), and the constant term is $m(\mathrm{i}, j, n)$ (here $b=0)$. Thus $b$ in this context is a parameter of nonorientability.

Table 1 of the Appendix gives the values of $m_{1 /(b+1)}(i, j, n)$ for maps with at most three edges. For example, the number 4 in (2) of Section 1 is consistent with the value $m((0,0,0,1), 1,2 ; b)=1+b+3 b^{2}$ in the table. The constant term 1 identifies the single orientable rooted map (in the torus) with 2 edges, a single face and a single vertex. The other terms $b$ and $3 b^{2}$ mean that there are indeed 4 nonorientable rooted maps (in the Klein bottle) with 2 edges, 1 face and 1 vertex. In this case, these 4 maps would be further subdivided into two classes, of size 1 and 3 , with different values, 1 and 2 , respectively, of the unknown measure of nonorientability recorded by this parameter.

We conclude that $\gamma$, which interpolates between complex curves and real curves in the context of Euler characteristics, has a separate and classical existence in terms of the Jack parameter. It should be noted that, even in the formal study of Jack functions themselves (rather than, for example, the complicated summation we are considering here), a combinatorial interpretation of the Jack parameter has been sought over the last decade (see Hanlon [7] and Stanley [28]), with recent success reported by Lapointe and Vinet [19] and Knop and Sahi [17].

5.3. Schur symmetric and zonal polynomials. We have introduced symmetric functions here indirectly, for technical reasons. However, the special cases $\gamma=1 / 2,1$ of the series $M_{\gamma}$ give the map series $M, M^{O}$, respectively, from (12). Moreover, the special cases $\gamma=1 / 2,1$ of Jack functions with parameter $1 / \gamma$ are zonal polynomials, Schur symmetric functions, respectively (in the latter case there is also a known scalar introduced). Applying these specializations to the Jack function expression (24) therefore yields expressions with zonal polynomials and Schur symmetric functions, respectively. These expressions have been obtained directly by Jackson and Visentin [16] (for $\gamma=1$ ) and Goulden and Jackson [6] (for $\gamma=1 / 2$ ). (This means that (23) and (24) have been proved in the cases $\alpha=2,1$ and $\gamma=1 / 2,1$, respectively.) The combinatorial encodings providing these direct derivations is quite different from the Wick's lemma methodology that yielded the integral expressions (9) for $M$ and (8) for $M^{O}$, in [5] and [14], respectively. We conclude with a sketch of these combinatorial encodings. For more complete details see the above references.

A graph embedded in an orientable surface is rooted, and the two ends of its $n$ edges are labelled from 1 to $2 n$, with the root end of the root edge labelled 1 . The map is encoded by two permutations in $\mathfrak{S}_{2 n}$. In the first, the disjoint cycles are the cyclic lists of the labels at the ends of edges incident with a vertex, in clockwise order, one for each vertex. In the second, the cycles are transpositions interchanging the labels at the two ends of a vertex, one for each edge. The cycles of the product of these permutations then specify the labels of edges in the boundary of each face of the map, and the determination of $M^{O}$ is therefore reduced to a computation in the centre of the group algebra of $\mathfrak{S}_{2 n}$ ), derivable from the connection coefficients of this subalgebra. The generating series for these yields the Schur symmetric function expression that follows from the summation over $\theta$ in (24) by setting $\gamma=1$. The 
logarithm is applied by the standard combinatorial construction to recover the connected components in this enumeration (since an arbitrary pair of permutations in the group algebra corresponds in general to an unordered collection of labelled maps). The operator $2 z \partial / \partial z$ is applied to account for the rooting of the map.

For $M$, a graph embedded in a locally orientable surface has the four side-end positions of its $n$ edges labelled from 1 to $4 n$, with the root side and end of the root edge labelled 1. The map is encoded by three matchings (pairings) on the $4 n$ symbols. In the first, the pairs consist of the labels that appear at the two ends of the same side of an edge. In the second, the pairs consist of the labels that appear at the two sides of the same end of an edge. In the third, the pairs consist of the labels that appear in a corner of a face. It can then be shown that the determination of $M$ can therefore be reduced to a computation in the double coset algebra of the hyperoctahedral group embedded in $\mathfrak{S}_{4 n}$ as the centralizer of a fixed fixed point free involution. The generating series that follows from this yields the zonal polynomial expression that follows from the summation over $\theta$ in (24) by setting $\gamma=1 / 2$. The logarithm and application of $4 z \partial / \partial z$ occur as in the determination of $M^{O}$.

Note added in proof. A related formula for the Euler characteristic in the real case appears in [3].

Appendix A. TABle

TABLE 1 . The refined map numbers $m_{1 /(b+1)}(\mathrm{i}, j, n)$, for $n \leq 3$ edges.

\begin{tabular}{|c|c|c|c|c|c|c|}
\hline$n$ & $j$ & $\mathrm{i}$ & $m_{1 /(b+1)}(\mathrm{i}, j, n)$ & $n \quad j$ & $\mathrm{i}$ & $m_{1 /(b+1)}(\mathrm{i}, j, n)$ \\
\hline \multirow[t]{3}{*}{1} & 1 & $(2)$ & 1 & \multirow[t]{7}{*}{3} & $(0,3)$ & 1 \\
\hline & & $(0,1)$ & $b$ & & $(1,1,1)$ & 12 \\
\hline & 2 & $(0,1)$ & 1 & & $(0,0,2)$ & $9 b$ \\
\hline \multirow[t]{8}{*}{2} & 1 & $(2,1)$ & 2 & & $(2,0,0,1)$ & 9 \\
\hline & & $(0,2)$ & $b$ & & $(0,1,0,1)$ & $15 b$ \\
\hline & & $(1,0,1)$ & $4 b$ & & $(1,0,0,0,1)$ & $30 b$ \\
\hline & & $(0,0,0,1)$ & $1+b+3 b^{2}$ & & $(0,0,0,0,0,1)$ & $10+10 b+32 b^{2}$ \\
\hline & 2 & $(0,2)$ & 1 & 3 & $(0,0,2)$ & 4 \\
\hline & & $(1,0,1)$ & 4 & & $(0,1,0,1)$ & 6 \\
\hline & & $(0,0,0,1)$ & $5 b$ & & $(1,0,0,0,1)$ & 12 \\
\hline & 3 & $(0,0,0,1)$ & 2 & & $(0,0,0,0,0,1)$ & $22 b$ \\
\hline \multirow[t]{9}{*}{3} & \multirow[t]{9}{*}{1} & $(2,2)$ & \multirow{9}{*}{$\begin{array}{c}3 \\
b \\
2 \\
12 b \\
1+b+5 b^{2} \\
9 b \\
3+3 b+9 b^{2} \\
6+6 b+18 b^{2} \\
13 b+13 b^{2}+15 b^{3} \\
\end{array}$} & \multirow[t]{9}{*}{4} & $(0,0,0,0,0,1)$ & 5 \\
\hline & & $(0,3)$ & & & & \\
\hline & & $(3,0,1)$ & & & & \\
\hline & & $(1,1,1)$ & & & & \\
\hline & & $(0,0,2)$ & & & & \\
\hline & & $(2,0,0,1)$ & & & & \\
\hline & & $(0,1,0,1)$ & & & & \\
\hline & & $(1,0,0,0,1)$ & & & & \\
\hline & & $(0,0,0,0,0,1)$ & & & & \\
\hline
\end{tabular}




\section{ACKNOWLEDGEMENTS}

This work was supported by grants individually to IPG and DMJ from the Natural Sciences and Engineering Research Council of Canada, and to JLH from the National Science Foundation (DMS-9401611).

\section{REFERENCES}

[1] D.Bessis, C.Itzykson And J.B.Zuber, Quantum field theory techniques in graphical enumeration, Adv. Applied Math. 1 (1980), 109-157. MR 83j:81067

[2] P.Buser And M.SeppäLÄ, Symmetric pants decompositions for Riemann surfaces, Duke Math. J. 67 (1992), 39-55. MR 93i:32026

[3] L.Chekhov and A.Zabrodin, A critical matrix model for non-oriented string, Modern Physics Letters A, 6 (1991), 3143-3152. MR 92m:81199

[4] I.P.Goulden AND D.M.JAcKson, Connection coefficients, matchings, maps and combinatorial conjectures for Jack symmetric functions, Trans. Amer. Math. Soc. 348 (1996), 873-892. MR 96m:05196

[5] I.P.Goulden AND D.M.JACKSON, Maps in locally orientable surfaces and integrals over real symmetric matrices, Canad. J. Math. 49 (1997), 865-882. MR 99d:05042

[6] I.P.Goulden AND D.M.JACKson, Maps in locally orientable surfaces, the double coset algebra, and zonal polynomials, Canad. J. Math. 48 (1996), 569-584. MR 97h:05051

[7] P.J. HANLON, Jack symmetric functions and some combinatorial properties of Young symmetrizers, J. Comb. Theory (A) 47 (1988), 37-70. MR 90e:05008

[8] J.L.Harer, The cohomology of the moduli space of curves, "Theory of Moduli", E.Sernesi, ed., Lecture Notes in Mathematics, Springer-Verlag, Heidelberg, 1337 (1988), 138-221. MR 90a:32026

[9] J.L.HARER, The virtual cohomological dimension of the mapping class group of an orientable surface, Invent. Math. 84 (1986), 157-176. MR 87c:32030

[10] J.HARER And D.ZAGier, The Euler characteristic of the moduli space of curves, Invent. Math. 85 (1986), 457-485. MR 87i:32031

[11] S.Helgason, "Differential geometry, Lie groups and symmetric spaces," Academic Press, New York, 1978. MR 80k:53081

[12] G. 'т Hoofт, A planar diagram theory for string interactions, Nuclear Physics B 72 (1974), 461-473.

[13] C.ItzzyKSOn And J.B.Zuber, Combinatorics of mapping class groups and matrix integration, preprint.

[14] D.M.JACKson, On an integral representation for the genus series for 2-cell embeddings, Trans. Amer. Math. Soc. 344 (1994), 755-772. MR 94k:05071

[15] D.M.JACKSON, Counting cycles in permutations by group characters, with an application to a topological problem, Trans. Amer. Math. Soc. 299 (1987), 785-801. MR 88c:05011

[16] D.M. Jackson And T.I. Visentin, A character theoretic approach to embeddings of rooted maps in an orientable surface of given genus, Trans. Amer. Math. Soc. 322 (1990), 343-363. MR 91b:05093

[17] F.Knop AND S.SAHI, A recursion and a combinatorial formula for Jack polynomials, Invent. Math. 128 (1997), 9-22. MR 98k:33040

[18] M.Kontsevich, Intersection theory on the moduli space of curves and the matrix Airy function, Commun. Math. Phys. 147 (1992), 1-23. MR 93e:32027

[19] L.Lapointe AND L.Vinet, A Rodrigues formula for the Jack polynomials and the Macdonald-Stanley conjecture, Int. Math. Notices 8 (1995), 419-424. MR 96i:33018

[20] F.Lesage, V.Pasquier And D.Serban Dynamical correlation functions in the CalogeroSutherland model, Nuclear Physics, B435 [FS] (1995), 585-603. MR 95m:82038

[21] I.G.Macdonald, "Symmetric functions and Hall polynomials," Second Edition, Clarendon Press, Oxford, 1995. MR 96h:05207

[22] M.L.Menta, "Random matrices," Second Edition, Academic Press, San Diego, 1991. MR 92f: 82002

[23] A. Okounkov, Proof of a conjecture of Goulden and Jackson, Canad. J. Math. 49 (1997), 883-886. MR 99f:05117 
[24] R.C.Penner, Perturbative series and the moduli space of Riemann surfaces, J.Diff. Geometry 27 (1988), 35-53. MR 89h:32045

[25] A.SelBerg, Bemerkninger om et multiplet integral, Norsk Matematisk Tidsskrift 26 (1944), 71-78. MR 8:269b

[26] M.SEPp ̈̈LÄ, Complex algebraic curves with real moduli, J. reine u. angew. Math. 387 (1988), 209-220. MR 89j:14021

[27] M.SEPPÄLÄ AND R.SILHOL, Moduli spaces for real algebraic curves and real abelian varieties, Math. Zeit. 201 (1989), 151-165. MR 90k:14043

[28] R.P.Stanley, Some combinatorial properties of Jack symmetric functions, Advances in Math. 77 (1989), 76-115. MR 90g:05020

[29] K.Strebel, On quadratic differentials with closed trajectories and second order poles, J.Anal. Math. 19 (1967), 373-382. MR 37:407

[30] E.Witten, Two dimensional gravity and intersection theory on moduli space, Surveys in Diff. Geom. 1 (1991), 243-310. MR 93e:32028

Department of Combinatorics and Optimization, University of Waterloo, Waterloo, Ontario, Canada, N2L 3G1

E-mail address: ipgoulden@math.uwaterloo.ca

Department of Mathematics, Duke University, Durham, North Carolina 27708-0320

E-mail address: harer@math.duke.edu

Department of Combinatorics and Optimization, University of Waterloo, Waterloo, Ontario, Canada, N2L 3G1

E-mail address: dmjackson@math.uwaterloo.ca 